\title{
Sprache und Bild als Forschungsgegenstand in linguistischen Disziplinen (Bild-, Text-, Medien- linguistik und Stilistik)
}

Struktury językowo-wizualne są wszechobecne w tekstach drukowanych, audiowizualnych i cyfrowych. Od pewnego czasu język i obraz w komunikacji medialnej są przedmiotem gorących dyskusji i interpretacji, przede wszystkim w lingwistyce germanistycznej i brytyjskiej. Niniejszy artykuł zbiera dotychczasowe głosy w dyskusji nad modelami i perspektywami w subdyscyplinach językoznawczych, w ramach których badana jest intensywnie komunikacja językowo-wizualna (lingwistyka obrazu, tekstu i mediów oraz stylistyka). Dyscypliny te przedstawione są w perspektywie germanistycznej i uzupełnione o dotychczasowy dorobek badań polonistycznych.

Sprachlich-visuelle Strukturen sind in gedruckten, audiovisuellen und digitalen Texten allgegenwärtig. Seit einiger Zeit sind Sprache und Bild in der massenmedialen Kommunikation ein Gegenstand heißer Diskussionen und Interpretationen vor allem in der germanistischen und britischen Linguistik. Der vorliegende Beitrag sammelt die bisherige Diskussion über Ansätze und Perspektiven in den linguistischen Subdisziplinen, in denen man sich intensiv mit der sprachlich-visuellen Kommunikation auseinandersetzt: Bild-, Text-, Medienlinguistik und Stilistik. Diese Disziplinen werden aus germanistischer Sicht behandelt und um Erkenntnisse bisheriger polonistischer Studien ergänzt.

At present, lingual-visual structures are ubiquitous in printed, audio-visual and digital texts. Language and images are in mass media communication a subject of hot debate and discussion, especially in the German and British linguistics. This paper aims to recapitulate the approaches and perspectives in the linguistic sub-disciplines which study intensively the lingual-visual communication (image linguistics, text and media linguistics and stylistics). The outline focuses on German linguistics research, however the Polish investigations are also presented. 
Roman Opiłowski

\section{Einleitende Vorbemerkungen}

Seit der Wettbewerbsfrage Brauchen wir einen neuen Textbegriff? (FIX / ADAMZIK / ANTOS / KLEMM 2002) und dem anregenden Aufsatz von Schmitz (2005) Blind für Bilder. Warum sogar Sprachwissenschaftler auch Bilder betrachten müssen enden die Forschungsfragen der Pragmalinguistik nicht bei der sprachlichen Textkonstitution, sondern reichen immer häufiger weiter. Sie konzentrieren sich auch auf andere als nur rein sprachliche Textbausteine. Die familiäre, schulische, universitäre, berufliche und nicht zuletzt städtische Kommunikation ist von Texten überfüllt, die heute aber aus mehreren Zeichensystemen - Sprache, Bild, Ton - bestehen. Das Sprachliche ist in der Regel das dominante Funktions- und Formelement von Texten. Die semiotische Komplexität von Texten im aktuellen medialen Zeitalter ist inzwischen eine Selbstverständlichkeit: Bilder, Grafiken, Tabellen, Schriftarten und visuelle Textstrukturen sind konstitutive Textbausteine, die in ihrem Zusammenwirken kohärente Gesamttexte als Realisationen bestimmter Textsorten zustande bringen. Die Beteiligung einzelner semiotischer Elemente an einem Text kann unterschiedlich sein, wobei die mediale Umgebung, technische Möglichkeiten, die Kommunikationsform und -funktion und der Rezipientenkreis die wichtigsten Determinanten sind.

Das unbestrittene Vorhandensein und sogar die Dominanz der semiotisch komplexen Texte bleiben nicht ohne Einfluss auf die Linguistik. Strikt linguistische Perspektiven auf Texte (z.B. auf Werbetexte), die formal und funktional gesehen auch aus anderen Zeichen als nur sprachlichen bestehen, führen zu partiellen oder gar zu eingeschränkten Erkenntnissen. Um im Sinne der Grundlagenforschung zu wahren und vollständigen Forschungsresultaten bei der medial vermittelten Kommunikation zu kommen, etabliert sich allmählich im deutschsprachigen Gebiet die sog. Bildlinguistik (vgl. Kap. 3). Sie akzentuiert stärker als jede andere Disziplin die Notwendigkeit einer interdisziplinären und intermethodologischen Untersuchung von Texten. Erste relevante Grundlagen und Methodologien zur Erfassung von Sprache und Bild im kommunikativen Handeln stammen aus der traditionsreichen Linguistik. Andere Studien, wie z.B. die hier vorliegende, sind jedoch erforderlich, um immer noch bestehende Defizite (z.B. in der interkulturellen bzw. kontrastiven Erforschung von medial führenden Sprache-Bild-Texten) auszugleichen.

Anders als in der germanistischen Forschung hat die polnische Linguistik wie unten gezeigt werden soll - noch nicht hinreichend die Text- und Medienlinguistik der sprachlich-bildlichen Kommunikation rezipiert, obwohl sich 
erste Ansätze allmählich abzeichnen. Ein Problemkreis betrifft die terminologische Bezeichnung mancher Phänomene zwischen Sprache und Bild. Denn die Hinzufügung bzw. Erweiterung des linguistischen Forschungsfeldes um visuelle und mitunter auditive Zeichen bringt einen neuen Blick auf bestehende (linguistische) Größen mit sich. Der neue semiotische Rahmen, in dem diese Zeichenvielfalt untersucht wird, muss nun auf bestimmte Weise benannt werden (vgl. Kap. 2).

Das letzte, dritte Element und zugleich grundlegende Ziel dieses Textes ist es, deutsche und polnische Aktivitäten bestehender linguistischer Subdisziplinen darzustellen, die Sprache und Bild im entsprechenden disziplinären Gefüge thematisieren. Wie oben angemerkt, werden zwischen den deutschen und polnischen Interessen deutliche Differenzen sichtbar. So bietet sich auch hier die Gelegenheit an, das fortschreitende Interesse deutschsprachiger Linguisten und das sich langsam entfachende Engagement polnischer Linguisten an der Sprache-Bild-Kommunikation zu reflektieren.

\section{Sprache + Bild $+[\ldots]=$ TEXT}

Der Titel dieses Unterkapitels widersetzt sich zum Teil dem Schwerpunktthema des vorliegenden Convivium. Dies ist insofern nötig, damit bestimmte terminologische Missverständnisse ausgeräumt werden. In den meisten Gebrauchstexten machen Sprache und Bild einen Text aus (vgl. SCHNEIDER / STÖCKL 2011). Ein Bild ist genauso wie Sprache ein Bestandteil des Textes. Je nach der Textsorte bzw. Kommunikationsform finden sich im multimodal profilierten Gesamttext sprachliche Teiltexte (Über- und Unterschriften, Vorwort und Nachwort, Titel und Kommentar etc.) und grafisch-bildliche Teiltexte (Tabellen, Grafiken, Abbildungen, Fotos etc.). Da die gesprochene oder geschriebene Sprache zentrale Medien in der textuellen Kommunikation sind, können sie einen Text konstituieren, während grafisch-bildliche Elemente als Zeichen des visuellen Systems keine Texte sind. ${ }^{1}$ An jedem Text muss die Sprache beteiligt sein, damit man von einer kommunikativfunktionalen Textgröße sprechen kann.

Die mathematische Gleichung im Kapiteltitel spiegelt heutige Tendenzen in der Texterfassung wider. Weniger handelt es sich hier um eine Textdefinition, weil die heutige moderne Linguistik von allen starren Grenzen, insbeson-

1 Im Gegensatz dazu STÖCKL (2004:96f.). 
dere auf dem Feld dynamischer Sprach- und Textprozesse, fern ist ${ }^{2}$, sondern vielmehr um eine Perspektive als eine unter einigen. Sprache und Bild, mit all ihren Zeichenmodalitäten (geschriebene und gesprochene Sprache, statische und animierte Bilder), erweisen sich heutzutage als zentrale Kommunikationsmedien. In Anbetracht dieser Tatsache kommunizieren wir mittels Texten, die natürliche, d.h. rudimentäre Größen in der zwischenmenschlichen, über Medien vermittelten Kommunikation sind.

Viele vor allem publizistisch oder digital geprägte Textsorten und Kommunikationsformen bestehen aus Sprache und Bild. Es wäre jedoch falsch zu behaupten, dass die bisherige Erforschung der Textformate unter dem sprachlichen Blickwinkel nur partielle Resultate über Formen und Funktionen von Texten geliefert hat. Entscheidend ist dabei die quantitative und qualitative Beteiligung eines Bildteils im Textgefüge. Falls wir es mit einem Romantext oder Behördenbrief zu tun haben, bei denen visuelle Elemente, wie z.B. Textdesign, Schrifttyp und Schriftgröße, eher eine untergeordnete Rolle spielen, können wir mit dem linguistischen Textansatz und der dazu gehörigen Methodologie das kommunikativ-funktionale Gerüst völlig erfassen. Demgegenüber sind wir zunehmend mit Texten konfrontiert, die wir uns ohne Bilder kaum vorstellen und verstehen können: Internetseiten, Software und Betriebssysteme im Smartphone und Computer, Pressetexte (insbesondere Boulevardzeitungen), belletristische und Fachliteratur, einschließlich linguistischer Studien- und Fachbücher und deren Inhaltsdarstellung (Gliederung, Hervorhebungen, Farbeneinsatz), erleichtern und optimieren mit der SpracheBild-Koordination den Wissenstransfer.

Jedes Textgefüge sollten wir als ein potenziell offenes Raster mit den semiotisch belegbaren Stellen betrachten. Die Kommunikationsbedürfnisse - und genau genommen kommunikative Aufgaben, die durch kommunikative Formate in medialen Plattformen oder Kanälen zu bewältigen sind - determinieren die Wahl einer Kommunikationsform. Sprachliche und bildliche Zeichen, die durch die Einfügung in eine kommunikative Situation eines Gesamttextes zu Teiltexten werden, enthalten untergeordnete Subformen. So lassen sich z.B. im Medium der geschriebenen Sprache unterschiedliche Schrifttypen unterscheiden. Die Handschrift kann auf Geschlecht, Alter oder sogar Charakterzüge hinweisen, während andere drucktechnische Schrifttypen für gan-

2 Den letzten umfassenden und sehr gewinnbringenden Versuch einer (Neu-)Bestimmung des Textbegriffes hat die Linguistik mit dem Sammelbad von FIX / ADAMZIK / ANTOS / KLEMM (2002) erfahren. 
ze Verlage, Zeitschriften, Markenprodukte oder sogar historische Epochen charakteristisch sind (vgl. STÖCKL 2008; OPIŁOWSKI 2010). Zu den bildlichen Textressourcen gehören Bildtypen und -sorten (Zeichnung, Foto, Infografik), der Farbengebrauch oder die visuelle Darstellung der Textstruktur (Textdesign).

Neben sprachlichen und bildlichen Zeichensystemen sollte man heute auditive Zeichen nicht übersehen, für die im Kapiteltitel die Stelle mit eingeklammerten Auslassungspunkten vorgesehen ist. In erster Linie sind hierbei audiovisuelle Texte von Bedeutung, in denen alle drei Medien - Sprache, Bild und Ton - formal, semantisch und ästhetisch kooperieren. ${ }^{3}$ Je nachdem, wie einzelne mediale Varietäten genutzt werden, kommt es zur reziproken Integration semiotischer Kodes, die letztlich eine kommunikativ-funktionale Textkohärenz schaffen.

\section{Bildlinguistik}

Die dem Gebiet von Sprache-Bild-Texten unmittelbar naheliegende Disziplin ist die sog. Bildlinguistik. Man wird jedoch weithin vergeblich nach etablierten Ansätzen und Methodologien suchen. Denn die Bildlinguistik ist eine linguistische Disziplin im Prozess des Entstehens. Ihr Gegenstand und ihre Aufgaben werden zum ersten Mal im Sammelband von DiEKMANNSHENKE / KLEMM / STÖCKL (2011) umrissen:

Vielmehr meint ,Bildlinguistik` die Betrachtung der Bezüge zwischen Sprache und Bild in Gesamttexten und die Nutzung linguistischer Konzepte, Modelle und Methoden für die Beforschung des in vorwiegend massenmediale Texte integrierten Bildes. (KLEMM / STÖCKL 2011:9)

In der genannten Publikation finden sich zahlreiche Beiträge, die rein linguistische Ansätze, Termini und Methoden auf multimodale Texte anzuwenden versuchen. Erste Anstöße für die Beschäftigung mit Sprache und Bild im Rahmen einer Bildlinguistik gehen von STÖCKL (2004) aus, der in der Monografie Die Sprache im Bild - Das Bild in der Sprache aktuelle Einsatzbereiche, Strategien und Funktionen und nicht zuletzt Typen von sprachlichen und bildlichen Interaktionen ausführlich darstellt. Stöckl bedient sich allerdings nicht des Terminus ,Bildlinguistik', aber seine tiefen Einsichten in die sprachlich-bildlichen Textstrukturen und angebotenen Analysen haben viele

3 STÖCKL (2007) beschreibt das eigenständige und integrative Auftreten auditiver Zeichen (Musik und Geräusch) in der Hörfunkwerbung. 
Textlinguisten und Medienwissenschaftler zur Erforschung der Beziehungen von Sprache, Bild und sogar Ton angeregt. Mit dieser und anderen Arbeiten bereitet Stöckl entsprechende Grundlagen für die interkulturelle und kontrastive Sprache-Bild-Forschung vor, die bisher selten thematisiert wird.

Die erste Stelle, an der die Bezeichnung ,Bildlinguistik ‘ auftaucht, ist der Artikel von HELD (2008). Die Autorin betrachtet sprachlich-bildliche Texte aus pragmatischer Sicht, bedient sich dabei der Beispiele aus der Tourismuswerbung und teilt bildlinguistische Textstrukturen in formale und semantische Domänen ein (HeLD 2008:156f.). Obwohl Held die Bildlinguistik sensu stricto nicht definiert und keine analytischen Vorgaben formuliert, macht sie auf den Bedarf bildlinguistischer Forschung aufmerksam und regt zur Weiterentwicklung dieser Disziplin an. Von der formalen und semantischen Gliederung von Held inspiriert schlage ich selbst in OPIŁOWSKI (2011) am Beispiel der Online- und Werbetexte einige Spezifizierungen vor. Zu den dominanten formalen Aspekten zähle ich die interne Textstruktur, formale Bildhaftigkeit und Typografie, während die Bildhaftigkeit und Remotivierung anschaulicher Formulierungen sowie Sprache-Bild-Beziehungen zu den inhaltlichen Aspekten gehören (OPIŁOWSKI 2011:199-208). Das sprachlichvisuelle Textdesign, das sich in letzter Zeit eines steigenden wissenschaftlichen Interesses erfreut (vgl. ROTH / SPITZMÜLLER 2007), erweist sich dabei als ein Bindeglied zwischen den beiden Domänen. Eine konstante und immer wieder in Texten wiederholte visuelle, thematische und strategische Textkonvention kann aus globaler Sicht als Textdesign betrachtet werden. Im Zeitalter der Informationsüberflutung gilt das Textdesign als das wirksame Persuasionsmittel zur schnellen Erkennung einer Werbekampagne, eines Pressetitels oder eines Internetportals.

Während die bisher skizzierten Arbeiten pragmatische Interessenfelder der Bildlinguistik anzeigen, befassen sich weitere Bearbeitungen auch mit theoretischen Ansätzen. Der Sammelband von DieKmannshenke / Klemm / STÖCKL (2011) macht das erste Mal die Bildlinguistik zum Ausgangspunkt der Überlegungen vieler Linguisten und Medienwissenschaftler. Im ersten, theoretisch-methodischen Buchteil widmen sich KLEMM / STÖCKL (2011) den Anfängen der Bildlinguistik, deren Ursprung im ,iconic turn' (BOEHM 1994) und ,picturial turn` (MitcheLL 2008) betrachtet wird. ${ }^{4}$ Vor diesem

$4 \quad$ Der ,iconic turn', den man als ein Forschungsprogramm auffassen kann, wird von Bовнм (1994) vorgeschlagen und bedeutet eine Erweiterung philosophischer, philologischer und künstlerischer Forschungsfelder um visuelle Semiosen. Der ,picturial turn', eingeführt von MitcheLl (2008), versteht sich als eine Art 
Hintergrund halten KLEMM / STÖCKL (2011:9) Forschungsdesiderate der Bildwissenschaften fest:

Meist ist die Rede von dem Bild. Dabei wird zu oft die Typisiertheit von Bildern und deren Vielfalt von Formen und Funktionen im sozialen Kontext übersehen. Statt kategorielle und pauschalisierende Aussagen über Bilder benötigen wir aber Erkenntnisse über konkrete Bildtypen und deren kommunikative Verwendung in fest umrissenen Situationen und Gebrauchsdomänen.

Diese erste Forschungslücke steht sehr nah an einer weiteren, die sich auf Bilder als von anderen Kommunikationszeichen isolierte Untersuchungsobjekte bezieht. Das dritte Forschungspostulat besagt, dass Bilder nicht als einzige Instrumente zum Erklären komplexer Erkenntnisprozesse gebraucht werden können. Der Perzeption, Rezeption, Manipulation oder Emotion lässt sich nämlich nicht nur durch Visualisierungen der Außenwelt nachkommen. Wenn sich die Bildwissenschaften das Aufschlüsseln dieser kognitiven Prozesse zum Ziel setzen, sollten sie dem ganzen semiotischen (also auch sprachlichen) Umfeld der gegebenen Kommunikationssituation Rechnung tragen.

Die System- und angewandte Linguistik ist dabei auch nicht ,ohne Schuld“. Bilder und ihre Variationen wurden in linguistischen Untersuchungen und Analysen lange Zeit als fakultativer Zusatz betrachtet, obwohl sie oft als ein gleichrangiges und evidentes Mitelement in einer bestimmten Kommunikationssituation existierten. So weist SCHMITZ (2005) sehr treffend auf Unzulänglichkeiten der angewandten Linguistik gegenüber der Bildkommunikation in der sprachlichen Umgebung hin.

KLEMM / STÖCKL (2011) skizzieren also ambitiöse Herausforderungen für die Bildlinguistik. Das ist ein Forschungsprogramm, das nicht nur machbar, sondern vor allem im Lichte der im Alltag steigenden multimodalen und -medialen Kommunikation und deren gesellschaftlicher Relevanz unentbehrlich ist. Vor einiger Zeit wurde die sprachlich-bildliche Kommunikation von Textproduzenten und -rezipienten als neu, modern und überraschend empfunden. Aktuell ist sie aus unterschiedlichen Perspektiven komplex geworden und gilt so als ein Standardfall der informativ-appellativen Kommunikation. In dieser Situation ist die Integration von Disziplinen und Forschungsmetho-

Ergänzung bzw. Fortsetzung des ,iconic turn` und konzentriert sich auf das Bild als eine visuelle Analogie zur Außenwelt. Das Bild wird zur Macht, spiegelt Schlüsselmomente von Weltereignissen wider und schafft samt der Sprache kommunikative Diskurse, die das Denken und Handeln von Kulturgemeinschaften erheblich beeinflussen. 
den notwendig, um die Fülle solcher Kommunikationsprozesse erklären zu können.

Wie sich die Bildlinguistik seit 2011 dynamisch entwickelt, bezeugt eine weitere umfassende Arbeit von GROßE (2011), die sich unmittelbar der BildLinguistik widmet. ${ }^{5}$ Diese Monografie reflektiert kaum Gegenstände, Ziele und Herausforderungen der Bildlinguistik, sondern thematisiert mit beinahe mathematischer Genauigkeit viele populäre Sprach- und Textansätze und versucht, sie auf Bildkomplexe zu übertragen. Der unanfechtbare Vorteil dieser Untersuchung liegt dennoch in der Revision linguistischer Phänomene und ihrer Brauchbarkeit für den Zugang zu Sprache-Bild-Texten. Textualitätskriterien, Sprechakttheorie oder linguistische Themenentfaltungen werden auf sprachlich-bildliche Texte bezogen. Insgesamt liefert GROßE (2011) eine theoretische sowie empirische linguistische Bildanalyse, wie es der Buchtitel ansagt, und weniger eine problematisierende Auseinandersetzung mit den mehrdimensionalen Abhängigkeiten von Sprache und Bild.

Die umrissenen Arbeiten und Probleme der aktuellen Bildlinguistik legen den raschen Bedarf an einer weiteren Entfaltung dieser sich formierenden Disziplin der Linguistik offen. Allgegenwärtige, mit Sprache und Bild überfüllte Kommunikationsformen und deren immer reichere Funktionsvielfalt sollten im interdisziplinären Forschungsfeld mit dem Schwerpunkt in der Sprache untersucht werden. So sind auf Medienspezifika, Merkmale von Textsorten und soziokulturelle Faktoren gestützte Ansätze, Typologien und Methoden erforderlich, damit man das Funktionieren und das Wirken von Sprache-BildKomplexen erkennen und entsprechend operationalisieren kann. Danach sind auch interkulturelle Studien möglich.

\section{Textlinguistik}

Erste theoretische und empirische Entwicklungsimpulse für die Bildlinguistik stammen aus zwei linguistischen Disziplinen, der Textlinguistik und der Stilistik, in denen man in den letzten Jahren ein steigendes Interesse an der grafisch-ikonischen Umgebung der Sprachtexte beobachtet. Die multimodale Textauffassung ist trotzdem in der funktional-kommunikativen und kognitiven Phase der Textlinguistik gar nicht selbstverständlich. Vielmehr versteht

5 Die unterschiedliche Schreibweise ist hier nicht zufällig. 
sie sich als eine Alternative zum ,klassischen Textbegriff` und wird je nach der Kommunikationsform entsprechend berücksichtigt. ${ }^{6}$

An der Informations-, Appell- und Unterhaltungsfunktion orientierte Texte, die mengenmäßig unseren Alltag am meisten überfluten, erweisen sich dabei als kreative und semiotisch dynamische Konstruktionen. Im Bemühen um die Gunst und Akzeptanz der Textempfänger müssen sie die perzeptiven und rezeptiven Gewohnheiten inhaltlich und formal brechen, vom Kanon üblicher Textgestaltung abweichen, um das Augenmerk der fokussierten Zielgruppe auf eine bestimmte Textbotschaft im Umfeld vieler anderer konkurrierender Textangebote zu fesseln. Werbung, Plakat und Pressetext, in denen die Rolle des Bildlichen kontinuierlich zunimmt, geben den ersten Anstoß zur multimodalen Textperspektive. ${ }^{7}$ Die enge Verflechtung von sprachlichen und bildlichen Textkonstituenten samt ihren weiteren semiotischen Bestandteilen (Design, Grafik, Typografie, Farben, Träger) veranlasst zu einem semiotisch holistischen Blick auf Textstruktur und -handeln. Eine solche Herangehensweise an den Text findet ihre legitime Anwendung bei digitalen Texten wie in Online-Zeitungen und sonstigen -Portalen, E-Mails und Weblogs, einschließlich Internetwerbung (vgl. SIEVER / SCHLOBINSKI / RUNKEHL 2005; FRAAS / MEIER / PENTZOLD 2012).

In der polonistischen Textlinguistik / Textologie, die entscheidend weniger an der Multimodalität von Texten interessiert ist, redet man von multimedialen Texten, während die germanistische Textlinguistik die Bezeichnungen multimodale Texte bzw. Kommunikate vielerorts definiert (vgl. SCHNEIDER / STÖCKL 2011). Das Problem liegt im unterschiedlichen Verstehen des Begriffes ,Medium', das man in der deutschsprachigen Textlinguistik entweder als semiotischen Kode oder als technologisch-technisches Instrument zum Vermitteln und Archivieren von Texten versteht. In der polonistischen

6 Gewisse Ausnahmen lassen sich bei den kognitiv orientierten Textlinguisten beobachten. So betont z. B. STROHNER (1995:2) die sprachliche und außersprachliche Textdimension, denn sie ist „nicht nur die sprachliche Information allein, sondern die Verbindung aller zu einem bestimmten Zweck produzierten Informationen (z. B. Sprache, Abbildung, Symbol) in einem oder mehreren Medien. Texte dienen dazu, bei den Rezipienten Wissen, Einstellungen und Emotionen hervorzurufen."

7 Vgl. STÖCKL (2004); FIX (2001); SCHIERL (2001); ECKKRAMMER / HELD (2006); JANICH (2010); SCHNEIDER / STÖCKL (2011); GRÖSSLINGER / HELD / STÖCKL (2012). 
Textforschung dominiert demgegenüber die alltagssprachliche, zeichenbezogene Verwendung des Mediums:

Multimediale Texte sind Texte, die mit der Anteilnahme von zwei oder mehreren semiotischen Kodes gebildet werden: dem sprachlichen oder auditiven Kode, wie auf Kassetten mit Gedichtrezitationen oder Liedern, dem sprachlichen und bildlichen Kode, wie in Büchern mit Abbildungen oder in Comics, dem auditiven und bildlichen, wie im Theater oder im Film [...]. (BARTMIŃSKI / NIEBRZEGOWSKA-BARTMIŃSKA 2009:71; Übersetzung - R. O.)

Ferner unterstreichen BARTMIŃSKI / NIEBRZEGOWSKA-BARTMIŃSKA (2009: 71f.) die Bedeutsamkeit von Hypertexten und der ikonischen Wende, wobei sie sich dennoch auf den Text aus sprachlicher Sicht einschränken. Eine der möglichen Erklärungen für diesen Stand der Dinge ist in der Forschungstradition zu sehen. Die polnische Textlinguistik konzentriert sich permanent auf drei linguistische Säulen: Dialekte, Metatexte und literarische Textsortenforschung. Der kommunikative Alltag, Gebrauchstexte, der massenmediale Sprachgebrauch finden mehr Beachtung bei polnischen Medienwissenschaftlern und Journalisten als bei Polonisten.

Demgegenüber kann man auf dem deutschsprachigen Gebiet von der sog. multimodalen Textlinguistik reden. Den ersten Anstoß dazu geben FIX / ADAMZIK / ANTOS / KLEMM (2002). Die meisten Beiträge, die als Antworten auf die im Bandtitel gestellte Frage konzipiert sind, plädieren für die Berücksichtigung visueller und verbaler Phänomene im Gesamtphänomen Text (vgl. z.B. MÖLLER 2002:96; KLEMM 2002; ADAMZIK 2002:173), um ganzheitliche und kohärente Ergebnisse der Textanalyse erhalten zu können. Jedoch, inwieweit und mit welcher Relevanz dies erfolgen soll, dabei gehen die Meinungen schon auseinander. Manche Forscher bemühen sich um eine unmittelbare Antwort auf die Titelfrage und postulieren unterschiedliche Sichtweisen auf den Text: der „erweiterte kommunikationsorientierte Textbegriff“ (ECKKRAMMER 2002:32), der „kulturwissenschaftliche Textbegriff“ (BREUER 2002:69) oder der „reflexionsdynamische Textbegriff“ (ANTOS 2002:189). Andere verankern auch den Textbegriff in der meta- bzw. transtextuellen Diskursivität (WARNKE 2002:125). ${ }^{8}$ Perspektivisch stellt ECKKRAMMER (2002:33) folgende Überlegungen an:

8 In diesem Zusammenhang muss man sich wiederum auf die darauf folgende imponierende Studie von STÖCKL (2004:96f.) beziehen, der nicht nur eine linguistisch fundierte Textualität des Bildes aufzeigt, sondern in der großzügigen Begeisterung für visuelle Kommunikation das Bild als Text auffasst. 
Die punktuelle Anpassung des Textbegriffs ist darüber hinaus auch aus dem Kontext der Rechtfertigung einer Disziplin in ihrer Zeit zu verstehen. Würde nämlich die Textlinguistik eine Anpassung an die kommunikativen Gegebenheiten der Aktualität verweigern und ihren Textbegriff nicht adaptieren, wird sich die Forschungspraxis dieser Wissenschaftsgemeinschaft nicht mehr erheblich weiterentwickeln.

Die Widerspiegelung und Fortsetzung dieser Sichtweise auf die Textlinguistik finden wir einige Jahre später bei FIX (2008:31):

Zunehmend hat die Textlinguistik im Blick, dass der Textbegriff vom rein sprachlich bestimmten auf einen multikodalen erweitert werden muss. Texte existieren nie nur sprachlich, immer sind andere Zeichen an ihnen beteiligt, seien es Gestik, Mimik, Stimmführung oder [...] Bilder, Typographie, Papiersorte usw. Da alle diese Zeichen gemeinsam Sinn anbieten, da sie alle auf der Textoberfläche und in der Textumgebung etwas zu verstehen geben und wahrgenommen werden sollen, kann man an ihnen nicht vorbeigehen. So kann man nicht den einen Kode, den sprachlichen, aus dem Textkomplex herauslösen und an ihm den Sinn des Ganzen ablesen wollen [...].

Die germanistische Textlinguistik reagiert auf neue Herausforderungen in der Kommunikation rechtzeitig. Anders verhält es sich mit der polonistischen Textlinguistik. Nach wie vor mangelt es am ausdrücklichen Interesse an der multikodalen Textkonstitution, dennoch zeigen sich erste Umorientierungen. In einem Interview zu Stand und Perspektiven der Textlinguistik macht DOBRZYŃSKA (2009:30) auf einige Forschungsfragen aufmerksam:

Meine weiteren Forschungsdesiderate betreffen die Beschreibung hybrider Texte, die sprachliche Elemente mit nichtsprachlichen verknüpfen. Solche Texte, die Wörter, Bilder oder Töne zu einer Ganzheit verschmelzen, unterliegen den getrennten Textmustern und sind auf besondere Weise aufgebaut. Das Wissen über dieses Thema ist weiterhin sehr eingeschränkt. (Übersetzung - R. O.)

Dass die polonistische Textlinguistik immer noch einiges in der multimodalen Textbetrachtung aufarbeiten muss, zeigt allein eine voreingenommene Bezeichnung von Sprache-Bild-Texten als „hybride Texte“ (s. Zitat oben). Es bleibt zu hoffen, dass diese Forschungsdesiderate rasch nachgeholt werden.

\section{Stilistik}

Die Forschungen zum Stil in der Kommunikation sind eng mit der Textlinguistik korreliert (SANDIG 2006; FIX / POETHE / YOS 2001; STÖCKL 2004), wobei dieser Zusammenhang in den polonistischen Studien geringer akzentuiert wird. Die bereits in der textlinguistischen Forschung angeführte FIX (2008) soll in diesem Kontext noch einmal erwähnt werden, denn der Beitrag 
dieser Germanistin zur Berücksichtigung und Erforschung visueller Elemente im Stil eines Textes ist beeindruckend. Ohne Übertreibung kann man sagen, dass Ulla Fix mit einer Serie von Monografien, Herausgeberschaften und einzelnen Beiträgen die deutschsprachige Textlinguistik auf die alltägliche Kommunikation hin öffnete und somit eine pragmatische Wende in der Textlinguistik aktiv begleitet hat. Die Textlinguistik wurde zu einer Gebrauchsdisziplin, die in erster Linie alltägliche Textsorten samt ihren Inhalten und Funktionen fokussiert. FIX (1992, 1996, 1996a, 2001a) führt konsequent das pragmatisch-semiotische Stilkonzept für Gebrauchs- und fiktive Texte durch. Nach FIX (1992:193) bewirkt eine solche Sichtweise die Uminterpretation des gängigen Stilbegriffes:

Bejaht man also die Frage nach etwas, das sich nur scheinbar von selbst versteht, nach dem nonverbalen Anteil an der Kommunikation und speziell am Stil, so hat das Folgen. Folgen für den Stilbegriff, Folgen für das, was man als stilistischen Kode verstehen will, und Folgen für den Bestand an Methoden der Stilanalyse. Wir dürfen dann nicht mehr von Sprachstil sprechen und ihn auch nicht mehr meinen. Die Rede sollte vielmehr von Kommunikationsstil sein und so unsere Vorstellungen auf die Komplexität des Stils im kommunikativen Handeln richten.

Der Sprachstil tritt zugunsten des Kommunikationsstils zurück, denn er ermöglicht einen breiteren und ganzheitlichen Blick auf den Textstil. Nicht nur geschriebene Texte, sondern materielles Bild, stilistische Ikonizität der Textstruktur, Größe, Typ und Farbe der Schriftzeichen, interne Textarchitektonik, grafisch-bildliche Linien oder Hervorhebungen schaffen durch eine formal-semantische Koordination den multimodalen Stil (vgl. FIX 1996, 2001a), der die „sich auf der Oberfläche des Textes realisierende Gestalt“ annimmt (FIX 1996:111).

Eine solche Perspektive auf Stil zeigte bald Konsequenzen: In weiteren Studien führt Fix Begriffe ein wie Supertext (FIX 1996), Gestalten des Stils als Gestalten der Einheitlichkeit (FIX 1996a), Ästhetisierung des Stils (FIX 2001), Verstehen des Textstils als Verstehen des Denkstils (FIX 2001a), Anschaulichkeit im materiellen und sprachlichen Bild (FIX 2002). In diese Reihe neuer Blicke, Begrifflichkeiten und Herausforderungen an den Stilbegriff schreibt sich der Sammelband von JaKOBS / RothKegel (2001) Perspektiven auf Stil ein. Der Stil wird in drei Bereiche eingeteilt: semiotisch, sprachlich-kulturell und sozial-historisch. Zum semiotischen Stilwesen gehört die Kategorie des Stils als Sprachdesign (vgl. ANTOS 2001:62). Das visuelle Textgestalten in der Form von Abbildungen, der grafischen Texteinteilung, Farben und Linien sind nicht nur mit der Sprachebene gleichrangige 
Stildeterminanten, sondern beeinflussen aus globaler Sicht den formalen und inhaltlichen Wandel von alltäglichen Textsorten wie Produktverpackungen, digitalen Hypertexten, Zeitungsartikeln, Print- und Außenwerbung. Das Sprachdesign manifestiert sich also in der Visualität von Texten (grafematisch, grafisch und ikonisch). In einem noch breiteren Umfang als im visuellen situiert RothKegel (2001:78) die Kategorie des Stils, der eine Komponente des Designs ist. Dieses gilt wiederum als eine Verknüpfung des Gestaltens einer bestimmten Materie (z.B. Produktdesign, Modedesign, Textdesign etc.) und des semantischen Gehalts.

Den grundlegenden Antrieb zum multimodalen Stil geben gegenwärtige Technologien der Textherstellung, deren expandierende Anwendung in allen drei sprachmedialen Gebieten (gedruckt, audiovisuell, digital) sichtbar wird. Darunter liefert das stilistische Textgestalten in digitalen Hypertexten unzählige Belege des kreativen Umgangs mit dem Stil. In diesem Sinn widmet sich STORRER (2001:93) dem digitalen Textstil, indem sie visuelle Stilaspekte auf die Anordnung der sprachlich markierten Links und die Anwesenheit animierter und statischer Illustrationen zurückführt. Die Autorin führt jedoch keine Diskussion über semantisch-funktionale Verhältnisse, die sich aus der semiotischen Stilgestalt ergeben. Eine praktische Weiterentwicklung des Stilbegriffes sehen wir bei STÖCKL (2003:311), der bestimmte Ebenen und Kriterien der multimodalen Stilanalyse darlegt. Den analytischen Arbeiten legt der Autor den folgenden Gedanken zugrunde:

Sprachstil zu betrachten heißt im Falle vieler moderner Textsorten eben auch, dessen Verbindungen zu anderen Zeichensystemen und deren Funktionsweisen zu erhellen. Der zunächst weite, von Sprache abrückende Weg führt dabei wieder zur Sprache zurück und am Ende näher an sie heran, denn Bild, Musik, Geräusche, Stimme und Rede sowie Typographie und Layout gehören als integrale Bestandteile zu unserer realen Erfahrung und praktischen Handhabung von Sprache und Kommunikation im Alltag. (STÖCKL 2003:321)

Für diese Stilauffassung greift STÖCKL (2003) auf einen Werbespot zurück und beschreibt in Bezug darauf einzelne semiotische Kodes (Sprache, Bild, Musik), um anschließend auf gemeinsame Aktivitäten dieser Kodes (mediale Varianten von Sprache untereinander, Sprache-Bild, Bild-Musik, SpracheMusik) einzugehen. Im Endergebnis entsteht ein Raster mit analytischen Kriterien, die je nach der multimodalen Kommunikationsform erweitert oder gekürzt werden können. ${ }^{9}$ Für den multimodalen Textstil plädiert auch SAN-

9 Im Einzelnen sind das: Strukturbildung - Segmentierung/Syntaktisierung, Denotation - Konnotation/Assoziation, Wiederholen - Parallelisieren - Komplemen- 
DIG (2006). Zwar bedient sich SANDIG (1986) in ihrer Stilistik der deutschen Sprache höchstens des Terminus ,Bildlichkeit“ im Hinblick auf metaphorische und phraseologische Anschaulichkeit, aber in SANDIG (2006:407f.) finden wir ausgearbeitete Analysen von Gebrauchstexten, in denen das materielle Bild in Verbindung mit der geschriebenen Sprache die Kohärenz des Stils bildet. Diese kohärente Stilgestalt resultiert nicht nur aus sprachlichen Kommunikaten und visuellen Darstellungen, sondern aus der Visualisierung der Typografie, der Anordnung einzelner sprachlicher und visueller Textteile, also der internen textuellen Topografie. Zu einer solchen „visuellen stilistischen Textgestalt“ (SANDIG 2006:451) werden funktionale Verknüpfungstypen von Sprache und Bild gezählt, die in einen Gesamttext münden (vgl. SANDIG 2006:455f.).

Für das multimodale bzw. semiotische Stilkonzept ist also das Auftreten und Zusammenwirken sprachlicher und visueller Textkomponenten charakteristisch. Eine solche Herangehensweise an Stilfragen kennzeichnet dennoch vorwiegend germanistische Studien. Polonistische Arbeiten reflektieren indes eine „semiotisch-kulturelle Stilauffassung“ (ZDUNKIEWICZ-JEDYNAK 2008: 16), aber ein solcher Blick konturiert den Stil als eine „transphrastische Satzstruktur, in der ein geordnetes Wertsystem bestimmte Sprachelemente expliziert [...]“ (Übersetzung - R. O.). Die semiotisch-kulturelle Dimension betrifft demzufolge keine Beteiligung unterschiedlicher Zeichenressourcen, sondern Implikationen aus stilistischen Registern; z. B. drückt ein umgangssprachlicher Sprachstil eine praktische und direkte Einstellung des jeweiligen Schreibers oder Sprechers aus, ein Amtsstil bezweckt ein formales Verhältnis zwischen Kommunikationspartizipanten. Im Grunde genommen ist das also keine semiotisch-kulturelle, sondern eine anthropozentrische Stilauffassung schlechthin. Eine gewisse Umorientierung zum multimodalen Stil kann die geführte Diskussion über den Stil in Hypertexten, die piktorielle Schrift im Internet oder die Sprachbilder im Textstil herbeiführen (vgl. ZDUNKIEWICZJEDYNAK 2008:85, 93; WIERZBICKA / WOLAŃSKI / ZDUNKIEWICZ-JEDYNAK 2008:24).

tieren, Abbilden/Verweisen/Symbolisieren, Analogiebildung. (Vgl. STÖcKL 2003:319f.). 
Sprache und Bild als Forschungsgegenstand in linguistischen Disziplinen

\section{Medienlinguistik}

Jeder Text tritt in einem Medium auf. Das kann ein physisch-technischer Textträger (z. B. ein Papierblatt und Buch für gedruckte Texte oder Computer, Smartphone, Navigation GPS oder CD-ROM für digitale Texte) oder ein sozialer Zugangskanal als Verbreitungsraum von Texten sein (z. B. Internet, Presse, Hörfunk, Fernsehen). Medien vereinen also technische Geräte und institutionalisierte Kommunikationstechniken (vgl. SZCZĘSNA 2007:21). Im Bereich der sog. Neuen Medien haben wir es schon mit technisch komplexen Plattformen zu tun, die virtuelle Texte virtuell bestehen und archivieren lassen. Die Medienlinguistik konzentriert sich demnach auf möglichst alle Medienfaktoren, die das Handeln mit vornehmlich sprachlichen Texten in bestimmter Weise determinieren. Sie beschreibt also die sprachlichen Phänomene in Abhängigkeit von ihrer Verwendung in einem Medium. STÖCKL (2012:14) räumt vor diesem Hintergrund ein:

Eine moderne Medienlinguistik darf aber niemals blind für den situativen Rahmen der Texte und ihre kulturellen Kontexte sein, sondern hat von ihnen auszugehen. Sie muss bewusst einklagen, dass letztlich nur ihre text- und sprachanalytische Methodik verlässliche Aussagen über die genaue Natur von Texten und Diskursen erlaubt.

Texte unterschiedlicher funktionaler Provenienz entstehen in und in der Verschränkung von Medien. Computeranwendungen gestatten, dynamische Änderungen in der Produktionsphase von digitalen Texten vorzunehmen. Sprache und Bild sind also aus formaler Perspektive unproblematisch miteinander zu verknüpfen, obwohl der Aspekt der funktional-semantischen Kohärenz damit ebenso einhergehen muss. Auf diese Umstände weist ScHMITZ (2004:112f.) hin:

Erst in dem Maße aber, wie Vierfarbdruck, Kino, Fernsehen und Computer es erlaubten, Bilder und Sprachzeichen auf derselben technischen Plattform gleich leicht zu (re)produzieren, wurde und wird die mediale und kulturelle Spannung zwischen Text und Bild ästhetisch komponiert und als Träger vielfältiger Botschaften genutzt. [...] Von Typographie, Layout und Textdesign über ,Illustrierte' und audiovisuelle Sendeformen (einschließlich Bildschirmteilung und -design, Computergrafiken und virtuellen Studios) bis hin zu Hypermedia ist der größte Teil massenmedialer Kommunikation (außer im Hörfunk) visuell aufwendig durchgestaltet.

Die Anwesenheit des Bildes und visuelle Darstellungen als immanente Komponenten polysemiotischer Texte werden von Medienwissenschaftlern und Philologen unterschiedlich akzentuiert. Obwohl jedes Medium ein Interaktionsgebiet der diversen Zeichen und in der Medienlinguistik eine grundlegen- 
de Rahmengröße ist, bedeutet dies nicht immer, dass andere Zeichen als gleichrangig mit den sprachlichen angesehen werden. Wenngleich die Forscher audiovisueller Texte an visuellen Bestandteilen nicht gleichgültig vorübergehen (vgl. ZWIEFKA-CHWAŁEK 2003; LUGINBÜHL 2011; HOLLY 2012), ist dies für gedruckte, informativ-publizistische Pressetexte nicht so selbstverständlich. Die letzten bieten im Unterschied zu den ersten zahlreiche sprachlich-bildliche Komplexe, können aber nur aus dem sprachlichen Text bestehen, so verständlich und kohärent sein. Andererseits sind z. B. Infografiken in der Presse ein hervorragendes Beispiel für ununterbrochene sprachlich-bildliche Relationen (vgl. ZWIEFKA-CHWAŁEK 2003:56-60; WOLNYZMORZYŃSKI / KALISZEWSKI / FURMAN 2009:41; STÖCKL 2012a). Sie kumulieren formal und semantisch ein Gefüge von Wort und Grafik, so dass beide wechselseitig determiniert sind. Eine solche Anhäufung und untrennbare Abhängigkeit von Wort und Grafik kann eine Konsequenz der Hypertextentwicklung sein, deren Tendenzen sich auch in der gedruckten Presse widerspiegeln (vgl. ZWIEFKA-CHWAŁEK 2003:56).

Ein weiteres Beispiel der konstanten Verwendung von Sprache und Bild in der Presse sind die ersten Titelseiten. Die meisten Pressemagazine enthalten spannungsvolle, mitunter rätselhafte Titelbilder, die erst nach dem Bezug auf Überschriften und Zwischentitel verständlich sind. Die deutsche Presse bedient sich auf den Titelseiten der persuasiven Strategie der Intertextualität und noch häufiger der Interikonizität (vgl. OPIŁOWSKI 2006, 2012). Zusammen mit den sprachlichen Kommentaren schaffen Titelbilder ein holistisches Textdesign (vgl. ANTOS / SPITZMÜLLER 2007; OPIŁOWSKI 2009), also eine spezifische Sehfläche (vgl. SCHMITZ 2011), die in ihrer visuell konstanten Ausformulierung einen bestimmten Pressetitel dauerhaft markieren und ihn so auch erkennen lassen. Im Medium Zeitung bzw. Magazin folgen den ersten Titelseiten publizistische Artikel, Feuilletons, Reportagen, Interviews, Horoskope, Wetterberichte, Leserbriefe etc. (vgl. WOJTAK 2004). Sie enthalten eben Fotos, Skizzen, Infografiken und satirische oder komische Geschichten in Bildern, d.h. die sog. Cartoons (vgl. STEGU 1987, 2000). So entstehen polysemiotische Texte. Selten beziehen sich jedoch Sprachtexte in der Presse auf das Bild allein: In der Regel illustrieren, veranschaulichen und unterstützen Pressebilder den Gedankengang und die Beschreibung und Argumentation im geschriebenen Pressetext. Demgegenüber wird die Dominanz der bildlichen Erzählform in der Fotoreportage (vgl. WOLNY-ZMORZYŃSKI / KALISZEWSKI / FURMAN 2009:62f.) deutlich. Eine Bilderfolge gibt einen bestimmten Wirklichkeitsausschnitt wieder. Sprachliche Texte definieren 
bzw. präzisieren dabei die Zeit, den Raum sowie den Ort des Bildgeschehens und benennen handelnde Akteure.

An der Grenze der journalistischen und nicht-journalistischen Texte situiert sich eine besondere Gruppe belletristischer Texte mit ludischer Funktion die Karikatur (vgl. HAMMER 2012; LENK 2012). Aus einigen Gründen erfreut sie sich eines Forschungsinteresses: In einem Magazin platziert, lockert sie manchmal den sachlichen journalistischen Berichtstil auf, ist politisch wirksam und hat einen unterhaltenden Charakter. In der Mehrheit der regionalen und überregionalen Presse in Europa (mit einem besonderen Akzent auf Italien und Frankreich) ist die Karikatur ein eigenständiges Element. Die aus der Karikatur fließende Reflexion und Konklusion sind erst durch die reziproke Bezugnahme des Bildes auf die Sprache und umgekehrt wahrzunehmen, obwohl bildzentrierte Karikaturen immerhin möglich sind. ${ }^{10}$ In der Studie zur deutschen, finnischen und britischen politischen Karikatur untersucht LENK (2012) die Kommunikationsebenen und bestehende Sprache-Bild-Bezüge. ${ }^{11}$ Als dominierende Form erweist sich das Comicmuster, während metakommunikative Bildkommentare sporadisch sind (vgl. LENK 2012:79). In dieser Zusammenstellung der journalistischen Textsorten in Sprache und Bild sollte die Werbung nicht fehlen. Sie kennzeichnet eine semiotische, formale, semantische und nicht zuletzt strategische Inszenierung, und so zieht sie die Aufmerksamkeit vieler Medienlinguisten an. Pragmalinguistische Gesamtblicke auf die Werbung finden sich in den Arbeiten von HELD / BENDEL (2008), STÖCKL (1997, 2004), SZCZĘSNA (2007) und JANICH (2010). Einzelne, sprachlich-visuelle Phänomene in der Werbung werden ebenso intensiv bearbeitet, wie z.B. Sprache-Bild-Verknüpfungstypen (vgl. SCHIERL 2001; STÖCKL 2004; OPIŁOWSKI 2006), persuasive Funktionen und Werbestrategien (vgl. STÖCKL 1997; JANICH 2010), intersemiotische Spannung (vgl. HELD 2006), Intertextualität in der Werbung (vgl. OPILOWSKI 2006) und der interkulturelle Dialog (vgl. HELD / BENDEL 2008).

Das Medium Buch ist der Erscheinungsort einer besonderen Sprache-BildSorte, der Comics. Eine deutliche Verwandtschaft weist der Comic mit der bereits erwähnten Fotoreportage und Karikatur auf. Von der ersten über-

10 Das jeweilige Bildgeschehen muss dann den einfachen Schlussregeln und Topoi folgen und das präsupponierte Bildwissen als Vorwissen kann nicht umfangreich und schwierig sein.

11 Im Falle der Sprache-Bild-Bezüge wendet Lenk (2012:76-81) die sprachlichvisuellen Gebrauchsmuster von STÖCKL (2004:253-300) an. 
nimmt er eine sequenzielle Weise der Darstellung, von der Karikatur eine fiktive Textwelt. Charakteristisch für den Comic ist die wechselseitige Determination als Sprache-Bild-Bezugstyp (vgl. OPIŁOWSKI 2006:118f.). Comics kennzeichnet nicht nur eine sprachlich-bildliche Verschränkung, sondern eine mehrdimensionale Funktionalität: informativ, ästhetisch und phatisch (vgl. KRIEGER 2003). Sie resultiert aus der durchaus gängigen Inszenierung der Schriftart und -größe, der Platzierung von Sprechblasen und Bildern, Farben und ihrer Sättigung.

Der urbane Raum enthält ebenfalls verschiedene Medien als Textträger. Die Wand, die in der prähistorischen Zeit in der Form einer Höhlenwand ein erster Bild- und Textträger war, erweist sich in jeder Klein- oder Großstadt als effektiver Bild- und Textträger künstlerischer Inhalte. Wände und Mauern sind also Medien für Graffiti. Um Botschaften und Emotionen zu vermitteln, schaffen (junge) Künstler typografische, ornamentale und figurale Bilder (vgl. MetTen 2011; Knieja 2011). Ikonisierte Texte begleiten Bilder und umgekehrt, so dass beide zu einer ästhetischen Gestalt und Einheit verschmelzen. Die sprachlich-visuelle Untrennbarkeit, Übergänge von Form und Inhalt sowie der rätselhafte Charakter der künstlerisch arrangierten Botschaft und des Appells sind einige Merkmale des Graffito als eines multimodalen Textes.

Das Internet ist ein Medium alter und neuer Kommunikationsformen und zugleich ein Ort unvorstellbarer Dynamik dieser Formen. Das Zusammenspiel von Sprache und Bild ist dabei mehr als offensichtlich, auch wenn sich das Verhältnis der Beteiligung von Sprache und Bild unterschiedlich gestalten kann. Komplexe Studien über Hypertexte in der Online-Kommunikation finden sich in SIEVER / SCHLOBINSKI / RUNKEHL (2005) und FRAAS / MEIER / PENTZOLD (2012). Im einleitenden Artikel der erstgenannten Publikation macht SCHLOBINSKI (2005:8) auf einige Tendenzen im Internet aufmerksam, unter denen er folgende aufzählt: „Die multimedialen Formate im Internet führen zu einer Neubestimmung der Funktion von Schrift und Bild: In zunehmendem Maße werden Objekte und Verhältnisse in der Welt durch (bewegte) Bilder dargestellt.“ Das ist eine Tendenz, die heutzutage nicht nur aktuell ist, sondern weiterhin an Dynamik zunimmt. In dieser Veröffentlichung gilt das Augenmerk einem weiteren Kapitel von Text und Bild im Focus, in dem RunKEHL (2005) die Sprache-Bild-Komplexe in Hypertexten vor dem Hintergrund zweier Faktoren - Multimedialität und Interaktivität reflektiert und SIEVER (2005) sich der Internetwerbung samt ihren sprachlichvisuellen Formaten und Inhalten widmet. 
Einen globalen Blick auf Sprache-Bild-Verhältnisse im Internet bietet ScHMITZ (2003:626), der von Koalitionen und Metamorphosen von Sprache und Bild zugleich redet. Der Bildschirm als Operationsfläche und die HyperlinkTechnik als Präsentationsmethode bewirken neue, in den anderen Medien nicht vorhandene Konstellationen von Sprache und Bild: „So ist eine neue und noch sehr dynamische Klasse von Text-Bild-Sorten entstanden, nämlich Lesebilder im Internet.“ (Hervorhebung - R. O.) Nicht nur gängige Internetseiten, sondern hypertextuelle Kommunikationsformen, die eine relativ unabgeschlossene Struktur, Funktion und einen entsprechenden Rezipientenkreis haben, können als spezifische Lesebilder betrachtet werden. Gemeint werden können an dieser Stelle Internetforen, E-Mails, Chats oder Weblogs, die Abbildungen, Grafiken, Emoticons oder ikonische Symbole enthalten (vgl. SchmiTz 2004). Dass neue Sprache-Bild-Sorten entstehen und unerwartet einen großen Gefallen bei Internetnutzern weltweit finden, beweisen die sog. Memes (vgl. SIEŃKO 2009). Dabei handelt es sich um wirklichkeitsnahe Bildmotive, die von einem oder mehreren Nutzern in Untertiteln zu diesen Bildern kommentiert werden. Witze, Ironie, komische Situationen, interikonische und -textuelle Bezüge sind bei Memes ein Standardfall.

Die Medienlinguistik als selbstständiges Fach oder Forschungsdisziplin wird in Polen (noch) nicht vertreten. Bei Internettexten und ihren Implikationen für Sprache und Bild zeigt sich hier unter allen Medien gleichwohl das größte Interesse polnischer Medien- und Kommunikationswissenschaftler sowie Journalisten (selten Philologen). Zwei wichtige Publikationen sind in diesem Zusammenhang ins Auge zu fassen: vor allem der Sammelband von FILICIAK / PTASZEK (2009), in dem Fragen der Online-Mediensprache, der Bildung durch das Internet und der Semiotik und Verwender in der OnlineKommunikation behandelt werden. Eine weitere Bearbeitung Tekst (w) sieci, tom 1 [Text (im) Netz, Bd. 1] von UliCKA (2009) widmet sich dem Text, der Sprache und den Textsorten in der Internetkommunikation. ${ }^{12}$ Die direkte Frage nach dem Status von Sprache und Bild in der Online-Kommunikation wird in den polnischen Beiträgen und selbstständigen Studien selten gestellt. Vielmehr wird die Sprach-Bild-Kommunikation am Beispiel bestimmter Online-Textsorten gleichsam gelegentlich berührt. So befassen sich ZWIEFKA-CHWALEK (2003), GRZENIA (2006), LOEWE (2006) und DATA (2009) mit den der verbalen Ebene gegenüber sekundär vertretenen Emoticons und an-

12 Dem Band 1 folgt Band 2, der aber nicht die linguistische, sondern die literaturwissenschaftliche, soziale und allgemein kommunikative Problematik der Internettexte fokussiert (vgl. GUMKOWSKA 2009). 
deren grafischen Darstellungen in Hypertexten. Die Internetforen mit ihren Avataren, Illustrationen und Emoticons interessieren SoKÓŁ (2009:207f.). Stark semiotisierte Videoblogs, die bewegte Bilder als textuelle Primärelemente enthalten, regen zur wissenschaftlichen Auseinandersetzung an (vgl. TURSKA-KAWA / ŚLAWSKA 2009:91-93). Die bereits erwähnten Memes sind neben der intensiven Sprache-Bild-Partnerschaft eine Herausforderung für Soziologen und Philosophen, weil sie, von Internetnutzern geschaffen, die sozial-kulturelle Realität kommentieren und verschlüsseln (vgl. SIEŃKO 2009). Eine Ausnahme ist der Beitrag von CHMIELECKI (2009), der sich unmittelbar den sprachlichen Texten in der Umgebung der Bilder und umgekehrt zuwendet. Er siedelt die Koexistenz von Sprache und Bild im Prozess des ,iconic turn' an und gibt dafür Beispiele von Flash-Animationen, erotischen Internetseiten, digitalen Fotografien oder Webkameras, die das performative Kommunizieren als Bildgeschehen realisieren. Wenig wird jedoch zu semiotischen, funktionalen und kommunikativen Aspekten der Sprache-BildRelationen ausgesagt.

Die Makrostruktur von Internettexten veranlasst WiTosz (2009:18) zu bestimmten Schlüssen über den Text als semiotisches Konstrukt:

Die Berücksichtigung der Multikodalität und Multimedialität des sprachlichen Kommunikats ist vom methodologischen Standpunkt her relevant. Dies erlaubt nämlich die Kategorie TEXT auf die multimediale Kommunikationssphäre auszudehnen [...], die ein immer aktiveres Interaktionsmodell ist. (Übersetzung R.O.)

Das obige Zitat beleuchtet und steckt auf eine richtige Weise den Weg des linguistischen Textes zum polysemiotischen ab. Die polonistische Textlinguistik sollte über das strikt umzäunte Terrain des sprachlichen Textes hinausschauen, inter- und transdisziplinär denken, weil die Offenheit der Termini und Wissenschaften keinen Verlust der Autonomie bedeutet, sondern zum Fortschritt einer Disziplin und zum effektiveren und vornehmlich wirklichkeitsnahen Erforschen der Artefakte beiträgt, wie sich dies in der germanistischen Textlinguistik ereignet: „Unter der Perspektive der Wissenschaftsgeschichte spricht die Reichhaltigkeit der Auseinandersetzung mit dem Textbegriff nicht gegen eine Textlinguistik, sondern für sie!“ (ANTOS 2002:89) 
Sprache und Bild als Forschungsgegenstand in linguistischen Disziplinen

\section{Literatur}

ADAMZIK, KiRSTEN (2002): Zum Problem des Textbegriffs. Rückblick auf eine Diskussion. In: Fix / ADAMZIK / ANTOS / KLEMm, 163-182.

ANTOS, GERD (2001): Sprachdesign als Stil. Lifting oder: Sie werden die Welt mit anderen Augen sehen. In: JAKOBS / ROTHKEGEL, 55-76.

- (2002): „Reflexionsdynamischer“ Textbegriff. Ein Blick in die Zukunft. In: FIX / ADAMZIK / ANTOS / KLEMM, 183-192.

ANTOS, GERD / SPITZMÜLLER, JÜRGEN (2007): Was bedeutet Textdesign? Überlegungen zu einer Theorie typographischen Wissens. In: RotH / SPITZMÜLLER, 35-48.

BARTMIŃSKi, JERZY / NiebRZEgOWSKA-BARTMIŃSKA, StANISŁAWA (2009): Tekstologia. [Textologie]. Warszawa.

Boehm, GotTFried (1994): Die Wiederkehr der Bilder. In: BoeHM, GotTFried (ed.): Was ist ein Bild? München, 11-38.

Breuer, Ulrich (2002): Wir schalten um. Text als Handlung/Text als Kommunikation. In: FIX / ADAMZIK / ANTOS / KLEMM, 59-71.

CHMIELECKI, KONRAD (2009): Tekst w sieci obrazów. Internet jako medium zapośredniczonej komunikacji wizualnej. [Der Text im Netz der Bilder. Das Internet als Medium der visuellen Kommunikation]. In: FILICIAK / PTASZEK, 298-313.

DATA, KRYSTYNA (2009): Wpływ komunikacji sieciowej na współczesna polszczyznę. [Der Einfluss der Online-Kommunikation auf das gegenwärtige Polnisch]. In: ULICKA, 131-138.

Diekmannshenke, Hajo / Klemm, Michael / StÖckl, HaRtmut (eds.) (2011): Bildlinguistik. Theorien - Methoden - Fallbeispiele. Berlin.

DoBRzYŃSKA, TERESA (2009): Lingwistyka tekstu w Polsce i w Niemczech - geneza, stan i perspektywy. [Textlinguistik in Polen und Deutschland - Genese, Stand und Perspektiven]. In: Bilut-Homplewicz, Zofia / Czachur, Waldemar / SMYKaŁa, Marta (eds.): Lingwistyka tekstu w Polsce i w Niemczech. Pojęcia, problemy, perspektywy. Wrocław, 17-30.

ECKKRAMMER, Eva MARTHA (2002): Brauchen wir einen neuen Textbegriff? In: FIX / ADAMZIK / ANTOS / KLEMM, 31-57.

ECKKrammer, Eva MARTHA / Held, GUdRun (eds.) (2006): Textsemiotik. Studien zu multimodalen Texten. Frankfurt (M.).

FiliciaK, MirosŁaw / PtAszeK, Grzegorz (eds.) (2009): Komunikowanie (się) w mediach elektronicznych. Język, edukacja, semiotyka. [Das Kommunizieren in elektronischen Medien. Sprache, Bildung, Semiotik]. Warszawa.

FIX, Ulla (1992): Stil als komplexes Zeichen im Wandel. Überlegungen zu einem erweiterten Stilbegriff. In: Zeitschrift für germanistische Linguistik 20.2:193-209.

- (1996): Textstil und KonTextstile. Stil in der Kommunikation als umfassende Semiose von Sprachlichem, Parasprachlichem und Außersprachlichem. In: FIX, UlLA / 
Lerchner, GotThard (eds.): Stil und Stilwandel. Bernhard Sowinski zum 65. Geburtstag gewidmet. Frankfurt (M.), 111-132.

- (1996a): Gestalt und Gestalten. Von der Notwendigkeit der Gestaltkategorie für eine das Ästhetische berücksichtigende pragmatische Stilistik. In: Zeitschrift für Germanistik VI-2:308-323.

- (2001): Die Ästhetisierung des Alltags - am Beispiel seiner Texte. In: Zeitschrift für Germanistik 1:36-53.

- (2001a): Zugänge zu Stil als semiotisch komplexer Einheit. Thesen, Erläuterungen und Beispiele. In: JAKOBS / ROTHKEGEL, 113-126.

- (2002): An-schauliche Wörter? Wörter im Dienste der ,Bildhaftigkeit‘, ,Bildlichkeit‘, ,Bildkräftigkeit', ,Sinnlichkeit', ,Lebendigkeit‘, ,Gegenständlichkeit‘ von Texten. In: Barz, Irmhild / Fix, Ulla / Lerchner, GotThard (eds.): Das Wort in Text und Wörterbuch. Stuttgart/Leipzig, 9-22.

- (2008): Text und Textlinguistik. In: JANICH, 15-34.

Fix, Ulla / Adamzik, Kirsten / Antos, Gerd / Klemm, Michael (eds.) (2002): Brauchen wir einen neuen Textbegriff? Antworten auf eine Preisfrage. Frankfurt (M.). Fix, Ulla / Poethe, Hannelore / Yos, Gabriele (eds.) (2001): Textlinguistik und Stilistik für Einsteiger. Ein Lehr- und Arbeitsbuch. Frankfurt (M.).

Fraas, Claudia / Meier, Stefan / Pentzold, Christian (eds.) (2012): OnlineKommunikation. Grundlagen, Praxisfelder und Methoden. München.

Große, FrANZISKA (2011): Bild-Linguistik. Grundbegriffe und Methoden der linguistischen Bildanalyse in Text- und Diskursumgebungen. Frankfurt (M.).

Grösslinger, Christian / Held, Gudrun / StÖcKL, Hartmut (eds.) (2012): Pressetextsorten jenseits der ,News‘. Medienlinguistische Perspektiven auf journalistische Kreativität. Frankfurt (M.).

GRZENIA, JAN (2006): Komunikacja językowa w Internecie. [Sprachliche Kommunikation im Internet]. Warszawa.

GumKowsKa, AnNa (ed.) (2009): Tekst (w) sieci. Literatura, społeczeństwo, komunikacja. [Text (im) Netz. Literatur, Gesellschaft, Kommunikation]. Bd. 2. Warszawa.

Hammer, Françoise (2012): Argumentation und Rekreativität der Pressezeichnung. Eine empirische Analyse der Karikaturen von Plantu in Le Monde. In: GrösSLINGER / HELD / STÖCKL, 53-64.

HELD, GUDRUN (2006): Formen intersemiotischer Spannung in aktueller Printwerbung. In: ECKKRAMMER / HELD, 107-128.

- (2008): Der Raum als Traum - ein Blick auf intersemiotische Gestaltungsstrategien und ihre Realisierung in globalen Kampagnen der Tourismuswerbung. In: HELD / BENDEL, 149-172.

HELD, GudRun / BENDEL, SylviA (eds.) (2008): Werbung - grenzenlos. Multimodale Werbetexte im interkulturellen Vergleich. Frankfurt (M.). 
HOLLY, WeRNER (2012): Bildüberschreibungen. Wie Sprechtexte Nachrichtenfilme lesbar machen (und umgekehrt). In: DiEKMANNSHENKE / KLEMM / STÖCKL, 233-253.

Jakobs, Eva-Maria / Rothkegel, Annely (eds.) (2001): Perspektiven auf Stil. Tübingen.

JANICH, NinA (1999 / ${ }^{5}$ 2010): Werbesprache. Ein Arbeitsbuch. Tübingen.

Klemm, Michael (2002): Ausgangspunkte: Jedem seinen Textbegriff? Textdefinitionen im Vergleich. In: FiX / ADAMZIK / ANTOS / KLEMM, 17-29.

Klemm, Michael / StÖCKL, Hartmut (2011): „Bildlinguistik“ - Standortbestimmung, Überblick, Forschungsdesiderate. In: DieKMANnSHENKE / KLEMM / STÖCKL, 7-18.

Knieja, Jolanta (2011): Urbane Graffiti aus textlinguistischer Sicht. In: Studia Niemcoznawcze. Studien zur Deutschkunde 47:565-575.

KRIEGER, JolAnTA (2003): Paraverbale Ausdrücke als Gestaltungsmittel der Textsorte Comic am Beispiel der Comic-Reihe Asterix. Lublin.

LenK, HaRTMut E.H. (2012): Politische Karikaturen in deutschen, englischen und finnischen Tageszeitungen. In: GRÖSSLINGER / HELD / STÖCKL, 65-81.

LOEWE, IWONA (2006): Internet i jego zasoby w polskich badaniach lingwistycznych. [Das Internet und seine Ressourcen polnischen linguistischen Untersuchungen]. In: Biuletyn Polskiego Towarzystwa Językoznawczego 62:93-103.

LUGINBÜHL, MARTin (2011): Vom kommentierten Realfilm zum multimodalen Komplex - Sprache-Bild-Beziehungen in Fernsehnachrichten im diachronen und internationalen Vergleich. In: DiEKMANNSHENKE / KLEMM / STÖCKL, 257-276.

Metten, Thomas (2011): Schrift-Bilder - Über Grafittis und andere Erscheinungsformen der Schriftbildlichkeiten. In: DieKMANNSHENKE / KLEMM / STÖCKL, 73-93.

Mitchell, William John Thomas (2008): Picture Theory: Essays on Verbal and Visual Representation. Chicago.

MÖLlER, LIOUdMila (2002): Beitrag zur Diskussion: Brauchen wir einen neuen Textbegriff? In: FiX / ADAMZIK / ANTOS / KLEMM, 93-96.

Opitowski, Roman (2006): Intertextualität in der Werbung der Printmedien. Eine Werbestrategie in linguistisch-semiotischer Forschungsperspektive. Frankfurt (M.).

- (2009): Das strategische Textdesign im Diskurs als Konstruktionsprinzip der Werbekampagnen. In: tekst i dyskurs. Text und Diskurs 2:107-121.

- (2010): Wie viel Bildlichkeit steckt in der Schriftlichkeit? Zur formalen und funktionalen Kreativität in der Werbetypographie. In: ŁOPUSZAŃSKA, GRAŻYNA (ed.): Sprache und Kultur als gemeinsames Erbe im Grenzgebiet. Sopot, 165-171.

- (2011): Bildlinguistik - Ansätze, Aspekte, Aufgaben. In: Colloquia Germanica Stetinensia 19:197-212.

- (2012): Interikonizität als Gestaltungsstil und Werbestrategie in Titelbildern. In: GRÖSSLINGER / HELD / STÖCKL, 37-51. 
Roth, Kersten Sven / SPITZMÜLLER, JÜRgen (eds.) (2007): Textdesign und Textwirkung in der massenmedialen Kommunikation. Konstanz.

RothKegel, ANnely (2001): Stil und/oder Design. In: JAKOBs / RothKegel, 77-87.

RunKeHL, Jens (2005): Text-Bild-Konstellationen. In: SIEVER / SCHLOBINSKI / RunKEHL, 202-218.

SANDIG, BARBARA (1986): Stilistik der deutschen Sprache. Berlin.

- (2006): Textstilistik des Deutschen. Berlin.

SchierL, Thomas (2001): Text und Bild in der Werbung. Bedingungen, Wirkungen und Anwendungen bei Anzeigen und Plakaten. Köln.

SCHLOBINSKI, PETER (2005): Sprache und internetbasierte Kommunikation - Voraussetzungen und Perspektiven. In: SIEVER / SCHLOBINSKI / RUNKEHL, 1-14.

SCHMITZ, UlRICH (2003): Lesebilder im Internet. Neue Koalitionen und Metamorphosen zwischen Text und Bild. In: Zeitschrift für Germanistik, Neue Folge XIII/3: 605-628.

- (2004): Sprache in modernen Medien. Eine Einführung in Tatsachen und Theorien, Themen und Thesen. Berlin.

- (2005): Blind für Bilder. Warum sogar Sprachwissenschaftler auch Bilder betrachten müssen. In: Osnabrücker Beiträge zur Sprachtheorie 69:187-227.

- (2011): Sehflächenforschung. Eine Einführung. In: DieKMANNSHENKE / KLEMm / STÖCKL, 23-42.

SCHNEIDER, JAN GEORG / StÖCKL, HARTMUT (eds.) (2011): Medientheorien und Multimodalität. Ein TV-Werbespot - Sieben methodische Beschreibungsansätze. Köln.

SieńKo, Marcin (2009): Demotywatory. Graficzne makra w komunikacji i kulturze. [Memes. Graphische Makros in der Kommunikation und Kultur]. In: FILICIAK, MiRoSŁAW / PTASZEK, GRZEGORz (eds.): Komunikowanie (sie) w mediach elektronicznych. Język, edukacja, semiotyka. Warszawa, 127-145.

SIEVER, TORSTEN (2005): Internetwerbung: Alter Wein in neuen Schläuchen!? In: SIEVER / SCHLOBINSKI / RUNKEHL, 219-241.

Siever, Torsten / Schlobinski, Peter / Runkehl, Jens (eds.) (2005): Websprache.net. Sprache und Kommunikation im Internet. Berlin.

SOKÓ£, MAŁGORZATA (2009): Repertuar podgatunków mowy forum internetowego $w$ perspektywie genologii lingwistycznej. [Zum Angebot von Textsortenvarianten in einem Internetforum in der Perspektive der Textsortenforschung]. In: ULICKA, 199-208.

STegu, MARTIN (1987): Der illustrierte Zeitungsartikel zwischen Semiotik und Textlinguistik. In: Semiotica Austriaca 9, 331-350.

- (2000): Text oder Kontext. Zur Rolle von Fotos in Tageszeitungen. In: FIX, UlLA / Wellmann, Hans (eds.): Bild im Text - Text und Bild. Heidelberg, 307-323. 
Sprache und Bild als Forschungsgegenstand in linguistischen Disziplinen

STÖCKL, HARTMUT (1997): Werbung in Wort und Bild. Textstil und Semiotik englischsprachiger Anzeigenwerbung. Frankfurt (M.).

- (2003): ,Imagine‘: Stilanalyse multimodal - am Beispiel des TV-Werbespots. In: BARZ, IRMHILD / LERCHNER, GotTHARD / SCHRÖDER, MARIANNE (eds.): Sprachstil Zugänge und Anwendungen. Heidelberg, 305-323.

- (2004): Die Sprache im Bild - Das Bild in der Sprache. Zur Verknüpfung von Sprache und Bild im massenmedialen Text. Konzepte - Theorien - Analysemethoden. Berlin/New York.

- (2007): Hörfunkwerbung - „Kino für das Ohr“. Medienspezifika, Kodeverknüpfungen und Textmuster einer vernachlässigten Werbeform. In: ROTH / SPITZMÜLLER, 177-202.

- (2008): Werbetypographie - Formen und Funktionen. In: HeLd / BeNDEL, 13-36.

- (ed.) (2010): Mediale Transkodierungen. Metamorphosen zwischen Sprache, Bild und Ton. Heidelberg, 145-172.

- (2012): Medienlinguistik. Zu Status und Methodik eines (noch) emergenten Forschungsfeldes. In: GRÖSSLINGER / HELD / STÖCKL, 13-34.

- (2012a): Finanzen visualisieren - Die Text-Bild-Sorte Infographik. In: Osnabrücker Beiträge zur Sprachtheorie 81:177-199.

StORRer, ANgelika (2001): Neue Medien - neue Stilfragen. Das World Wide Web unter stilistischer Perspektive. In: JAKOBS / ROTHKEGEL, 89-111.

STROHNER, HANS (1995): Kognitive Systeme. Eine Einführung in die Kognitionswissenschaft. Opladen.

SzCzęsna, Ewa (2007): Poetyka mediów. [Die Poetik der Medien]. Warszawa.

Turska-Kawa, AgnieszKa / Ślawska, Magdalena (2009): „Stowo się rzekło“. Analiza psychologiczno-lingwistyczna bloga Jerzego Bralczyka. [„,Versprochen ist versprochen“. Psychologisch-linguistische Analyse des Weblogs von Jerzy Bralczyk]. In: FILICIAK / PTASZEK, 79-95.

Ulicka, Danuta (ed.) (2009): Tekst (w) sieci. Tekst, język, gatunki. [Text (im) Netz. Text, Sprachen, Gattungen]. Bd. 1. Warszawa.

WARNKE, INGO (2002): Adieu Text - bienvenue Diskurs? Über Sinn und Zweck einer poststrukturalistischen Entgrenzung des Textbegriffs. In: FIX / ADAMZIK / ANTOS / KLEMM, 125-141.

WierZBickA, ElŻBieta / WOLAŃSKI, AdAM / ZDUNKIEWICZ-JEDYNAK, DOROTA (2008): Podstawy retoryki i stylistyki. [Grundfragen der Rhetorik und Stilistik]. Warszawa.

WitOsz, BożENA (2009): Lingwistyczne koncepcje tekstu wobec wyzwań komunikacji wirtualnej. [Linguistische Textansätze im Lichte der virtuellen Kommunikation]. In: ULICKA, 15-26.

WOJTAK, MARIA (2004): Gatunki prasowe. [Presse-Gattungen]. Lublin. 


\section{Roman Opiłowski}

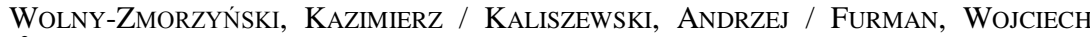
(2009): Gatunki dziennikarskie. Teoria - praktyka - język. [Textsorten im Journalismus. Theorie - Praxis - Sprache]. Warszawa.

ZdunKIEWICZ-JeDYNAK, Dorota (2008): Wyktady ze stylistyki. [Vorträge in Stilistik]. Warszawa.

ZwiefKA-ChwaŁeK, AgniesZKa (2003): Stowo elektroniczne. Strategie werbalne w epoce nowych mediów. [Das elektronische Wort. Verbale Strategien im Zeitalter neuer Medien]. In: WoźNY, ALEKSANDER (ed.): Translokacje i transpozycje w mediach. Wrocław, 15-67. 